\title{
On multiple higher Mahler measures and multiple $L$ values
}

by

$$
\text { Yoshitaka SASAKi (Osaka) }
$$

1. Introduction and notation. For every Laurent polynomial $P \in$ $\mathbb{C}\left[X_{1}^{ \pm 1}, \ldots, X_{n}^{ \pm 1}\right] \backslash\{0\}$, the (logarithmic) Mahler measure of $P$ is defined by

$$
m(P):=\int_{0}^{1} \cdots \int_{0}^{1} \log \left|P\left(e^{2 \pi i t_{1}}, \ldots, e^{2 \pi i t_{n}}\right)\right| d t_{n} \cdots d t_{1} .
$$

It is known that, in some cases, the Mahler measure relates to zeta or $L$ values, for instance, Smyth [6] showed

$$
m(1+X+Y)=m(1-X-Y)=\frac{3 \sqrt{3}}{4 \pi} L\left(2, \chi_{-3}\right),
$$

where $\chi_{-3}$ is the quadratic character attached to $\mathbb{Q}(\sqrt{-3})$ and $L\left(*, \chi_{-3}\right)$ is the Dirichlet $L$ series associated with a character $\chi_{-3}$. See [3], [4] and the references therein for other known cases.

Kurokawa, Lalín and Ochiai [5] introduced the $k$-higher Mahler measure of $P$ and the multiple higher Mahler measure of $P_{1}, \ldots, P_{l}$ defined by

$$
m_{k}(P):=\int_{0}^{1} \cdots \int_{0}^{1} \log ^{k}\left|P\left(e^{2 \pi i t_{1}}, \ldots, e^{2 \pi i t_{n}}\right)\right| d t_{n} \cdots d t_{1}
$$

and

$$
m\left(P_{1}, \ldots, P_{l}\right):=\int_{0}^{1} \cdots \int_{0}^{1} \prod_{q=1}^{l} \log \left|P_{q}\left(e^{2 \pi i t_{1}}, \ldots, e^{2 \pi i t_{n}}\right)\right| d t_{n} \cdots d t_{1},
$$

respectively. Here, $P$ and $P_{j}$ 's $(j=1, \ldots, l)$ are in $\mathbb{C}\left[X_{1}^{ \pm 1}, \ldots, X_{n}^{ \pm 1}\right] \backslash\{0\}$. In [5], Kurokawa, Lalín and Ochiai evaluated some examples of $k$-higher Mahler measure and the multiple higher Mahler measure and showed a relation between the multiple higher Mahler measure of $1-X$ and multiple

2010 Mathematics Subject Classification: Primary 11M32; Secondary 11M06.

Key words and phrases: Mahler measure, multiple zeta value, multiple $L$ value, MordellTornheim zeta value. 
zeta values:

$$
m_{k}(1-X)=(-1)^{k} k ! \sum_{h \geq 1} \frac{1}{2^{2 h}} \sum_{\begin{array}{c}
b_{1}, \ldots, b_{h} \geq 2 \\
b_{1}+\cdots+b_{h}=k
\end{array}} \zeta\left(b_{1}, \ldots, b_{h}\right),
$$

where $\zeta\left(b_{1}, \ldots, b_{h}\right)$ is the multiple zeta value defined by

$$
\zeta\left(b_{1}, \ldots, b_{h}\right):=\sum_{0<n_{1}<\cdots<n_{h}} \frac{1}{n_{1}^{b_{1}} \cdots n_{h}^{b_{h}}}
$$

for $\left(b_{1}, \ldots, b_{h}\right) \in \mathbb{N}^{h}$ with $b_{h} \geq 2$. They also proved

$$
m_{2}(1+X+Y)=\frac{5 \pi^{2}}{54}
$$

which is a generalization of the result (1.1) of Smyth. Further, Akatsuka evaluated $m_{k}(a-X)(a \in \mathbb{C},|a|<1)$, and showed in [1] that $m_{k}(a-X)$ can be written in terms of multiple polylogarithms.

In this article, we will evaluate the multiple higher Mahler measure $m\left(1-X-Y_{1}, \ldots, 1-X-Y_{k}\right)$ and see that those values relate to multiple $L$ values. However the type of multiple $L$ values in our case is slightly different from that of the multiple $L$ values treated by Arakawa and Kaneko [2]. Our result is another generalization of the result (1.1) of Smyth.

2. Statement of result. To state the main theorem, we have to introduce some notation.

We put

$$
w_{-3}(n):=\frac{(-1)^{n} \chi_{-3}(n)}{n}, \quad \widetilde{w}_{-3}(n):=n w_{-3}(n), \quad \text { with } \chi_{-3}(n):=\left(\frac{n}{3}\right) .
$$

Then we define multiple $L$ values by

$$
L\left(r_{1}, \ldots, r_{k} ; w_{-3} ; k, v\right)=\sum_{0<n_{1}<\cdots<n_{k}} \frac{w_{-3}\left(n_{k}-n_{k-v}\right)}{n_{1}^{r_{1}} \cdots n_{k}^{r_{k}}}
$$

for $v=1, \ldots, k-1$, and

$$
L\left(r_{1}, \ldots, r_{k} ; \widetilde{w}_{-3} ; k\right)=\sum_{0<n_{1}<\cdots<n_{k}} \frac{\widetilde{w}_{-3}\left(n_{k}\right)}{n_{1}^{r_{1}} \cdots n_{k}^{r_{k}}} .
$$

In particular, for the case of $k=2$, we have

$$
\begin{aligned}
L\left(r_{1}, r_{2} ; w_{-3} ; 2,1\right) & =\sum_{0<n_{1}<n_{2}} \frac{(-1)^{n_{2}-n_{1}} \chi_{-3}\left(n_{2}-n_{1}\right)}{n_{1}^{r_{1}} n_{2}^{r_{2}}\left(n_{2}-n_{1}\right)} \\
& =\sum_{m_{1}=1}^{\infty} \sum_{m_{2}=1}^{\infty} \frac{\widetilde{w}_{-3}\left(m_{2}\right)}{m_{1}^{r_{1}} m_{2}\left(m_{1}+m_{2}\right)^{r_{2}}},
\end{aligned}
$$


which is a "Mordell-Tornheim $L$ value" (for instance, see [7]). Therefore, if we put

$$
L_{\mathrm{MT}, 2}\left(s_{1}, s_{2}, s_{3} ; \widetilde{w}_{-3}\right):=\sum_{n_{1}=1}^{\infty} \sum_{n_{2}=1}^{\infty} \frac{\widetilde{w}_{-3}\left(n_{2}\right)}{n_{1}^{s_{1}} n_{2}^{s_{2}}\left(n_{1}+n_{2}\right)^{s_{3}}},
$$

then we can write $L_{\mathrm{MT}, 2}\left(r_{1}, 1, r_{2} ; \widetilde{w}_{-3}\right)=L\left(r_{1}, r_{2} ; w_{-3} ; 2,1\right)$.

TheOREm 2.1. For any positive integer $k$, we have

$$
\begin{aligned}
m(1- & \left.X-Y_{1}, \ldots, 1-X-Y_{k}\right) \\
= & \frac{(-1)^{k} k !}{3} \sum_{h \geq 1} 2^{1-2 h} \sum_{\begin{array}{c}
b_{1}, \ldots, b_{h} \geq 2 \\
b_{1}+\cdots+b_{h}=k
\end{array}} \zeta\left(b_{1}, \ldots, b_{h}\right) \\
& \quad+\frac{(-1)^{k} k ! \sqrt{3}}{2^{k} \pi}\left\{L\left(\{1\}_{k-1}, 2 ; \widetilde{w}_{-3} ; k\right)+\sum_{j=1}^{k-1}\left(T_{1, j}+T_{2, j}\right)\right\},
\end{aligned}
$$

where $\{1\}_{\mu}=(\underbrace{1, \ldots, 1}_{\mu})$ is a $\mu$-tuple,

$$
\begin{aligned}
\text { (2.4) } T_{1, j}= & \sum_{v=1}^{\kappa_{j}-1} \sum_{h=1}^{\min \left\{j, \kappa_{j}-v\right\}}\left(\begin{array}{c}
k-v-2 h \\
j-h
\end{array}\right) \\
& \times \sum_{\left(a_{1}, \ldots, a_{h}\right) \in \mathcal{S}_{h}^{k-v}} L(\underbrace{\{1\}_{a_{1}}, 2, \ldots,\{1\}_{a_{h}}, 2}_{k-v-h},\{1\}_{v} ; w_{-3} ; k-h, v)
\end{aligned}
$$

and

$$
\begin{aligned}
& \text { (2.5) } \quad T_{2, j}=\sum_{v=1}^{\kappa_{j}} \sum_{h=1}^{\min \left\{j, \kappa_{j}-v+1\right\}}\left(\begin{array}{c}
k-v+1-2 h \\
j-h
\end{array}\right) \\
& \times \sum_{\left(a_{1}, \ldots, a_{h}\right) \in \mathcal{S}_{h}^{k-v+1}} L(\underbrace{\{1\}_{a_{1}}, 2, \ldots,\{1\}_{a_{h-1}}, 2,\{1\}_{a_{h}}}_{k-h-v},\{1\}_{v+1} ; w_{-3} ; k-h+1, v),
\end{aligned}
$$

with $\mathcal{S}_{h}^{k}:=\left\{\left(a_{1}, \ldots, a_{h}\right) \in \mathbb{N}_{0}^{h} \mid a_{1}+\cdots+a_{h}=k-2 h\right\} ;$ here $\mathbb{N}_{0}:=\mathbb{N} \cup\{0\}$ and $\kappa_{j}=k-j$.

REMARK 2.2. When $h=1$, the last sum on the right-hand side of 2.5 is $L\left(\{1\}_{k} ; w_{-3} ; k, v\right)$.

Corollary 2.3. If we put $k=2$ in Theorem 2.1, then

$$
\begin{aligned}
m(1-X-Y, 1 & -X-Z) \\
& =\frac{1}{3} \zeta(2)+\frac{\sqrt{3}}{2 \pi}\left\{L\left(1,2 ; \widetilde{w}_{-3} ; 2\right)+L_{\mathrm{MT}, 2}\left(1,1,1 ; \widetilde{w}_{-3}\right)\right\} .
\end{aligned}
$$


3. Preliminary lemmas. The following two lemmas are important and essential tools for evaluating Mahler measures.

Lemma 3.1 (Jensen's formula). For $\alpha \in \mathbb{C} \backslash\{0\}$, we have

$$
\int_{0}^{1} \log \left|\alpha-e^{2 \pi i t}\right| d t=\log \max \{1,|\alpha|\} .
$$

Lemma 3.2 (Kurokawa, Lalín and Ochiai [5]). Let $x=e^{2 \pi i t}$ with $0<$ $t<1$. Then

$$
\log ^{k}|1-x|=\frac{(-1)^{k} k !}{2^{k}} \sum_{j=0}^{k} \operatorname{Li}_{\underbrace{1, \ldots, 1}_{j}}(x) \operatorname{Li}_{\underbrace{1, \ldots, 1}_{k-j}}\left(x^{-1}\right),
$$

where

$$
\operatorname{Li}_{r_{1}, \ldots, r_{k}}(x):=\sum_{0<n_{1}<\cdots<n_{k}} \frac{x^{n_{k}}}{n_{1}^{r_{1}} \cdots n_{k}^{r_{k}}}
$$

is the multiple polylogarithm.

4. Proof of Theorem 2.1. From the definition of the multiple higher Mahler measure and Lemma 3.1, we have

$$
\begin{aligned}
m(1-X & \left.-Y_{1}, \ldots, 1-X-Y_{k}\right) \\
& =\int_{0}^{1}\left\{\prod_{q=1}^{k} \int_{0}^{1}\left(\log \left|1-e^{2 \pi i t}-e^{2 \pi i t_{q}}\right|\right) d t_{q}\right\} d t \\
& =\int_{0}^{1}\left(\log \max \left\{1,\left|1-e^{2 \pi i t}\right|\right\}\right)^{k} d t=\int_{1 / 6}^{5 / 6} \log ^{k}\left|1-e^{2 \pi i t}\right| d t .
\end{aligned}
$$

The right-hand side of (3.1) can be split as

$$
\operatorname{Li}_{\underbrace{1, \ldots, 1}_{k}}(x)+\operatorname{Li}_{\underbrace{1, \ldots, 1}_{k}}\left(x^{-1}\right)+\sum_{j=1}^{k-1}\left\{D_{j}+2 \Re S_{j}(x)\right\}
$$

for $x=e^{2 \pi i t}(0<t<1)$, where

$$
\begin{aligned}
& D_{j}:=\sum_{\substack{0<l_{1}<\cdots<l_{j-1}<u \\
0<n_{1}<\cdots<n_{k-j-1}<u}} \frac{1}{l_{1} \cdots l_{j-1} n_{1} \cdots n_{k-j-1} u^{2}}, \\
& S_{j}(x):=\sum_{\substack{0<l_{1}<\cdots<l_{j} \\
0<n_{1}<\cdots<n_{k-j} \\
l_{j}<n_{k-j}}} \frac{x^{n_{k-j}-l_{j}}}{l_{1} \cdots l_{j} n_{1} \cdots n_{k-j}} .
\end{aligned}
$$


We can easily evaluate

$$
\int_{1 / 6}^{5 / 6} \operatorname{Li}_{\underbrace{1, \ldots, 1}_{1}}\left(e^{2 \pi i t}\right) d t=\frac{\sqrt{3}}{2 \pi} L\left(\{1\}_{k-1}, 2 ; \widetilde{w}_{-3} ; k\right)
$$

and

$$
\int_{1 / 6}^{5 / 6} D_{j} d t=\frac{2}{3} D_{j}
$$

since $\chi_{-3}(n)=2 \sin (2 \pi n / 3) / \sqrt{3}$. It is shown in [5] that

$$
\sum_{j=1}^{k-1} D_{j}=\sum_{h=1}^{k-1} 2^{k-2 h} \sum_{\left(a_{1}, \ldots, a_{h}\right) \in \mathcal{S}_{h}^{k}} \zeta\left(a_{h}+2, \ldots, a_{1}+2\right) .
$$

Note that the sum on the right-hand side of (4.4) vanishes for $h>[k / 2]$. Hence

$$
\sum_{j=1}^{k-1} \int_{1 / 6}^{5 / 6} D_{j} d t=\frac{2}{3} \sum_{h=1}^{k-1} 2^{k-2 h} \sum_{\left(a_{1}, \ldots, a_{h}\right) \in \mathcal{S}_{h}^{k}} \zeta\left(a_{h}+2, \ldots, a_{1}+2\right) .
$$

Similarly,

$$
\begin{aligned}
& \int_{1 / 6}^{5 / 6} \Re S_{j}\left(e^{2 \pi i t}\right) d t \\
& =\frac{\sqrt{3}}{2 \pi} \sum_{\substack{0<l_{1}<\cdots<l_{j} \\
0<n_{1}<\cdots<n_{k-j} \\
l_{j}<n_{k-j}}} \frac{(-1)^{n_{k-j}-l_{j}} \chi_{-3}\left(n_{k-j}-l_{j}\right)}{l_{1} \cdots l_{j} n_{1} \cdots n_{k-j}\left(n_{k-j}-l_{j}\right)}=\frac{\sqrt{3}}{2 \pi} \widetilde{S}_{j} .
\end{aligned}
$$

We split $\widetilde{S}_{j}$ as follows:

$$
\begin{aligned}
\widetilde{S}_{j} & =\sum_{v=1}^{\kappa_{j}-1} \sum_{\substack{0<l_{1}<\cdots<l_{j} \\
0<n_{1}<\cdots<n_{\kappa_{j}} \\
l_{j}=n_{\kappa_{j}-v}}}+\sum_{v=1}^{\kappa_{j}} \sum_{\begin{array}{c}
0<l_{1}<\cdots<l_{j} \\
0<n_{1}<\cdots<n_{\kappa_{j}} \\
n_{\kappa_{j}-v}<l_{j}<n_{\kappa_{j}}-v+1
\end{array}} \\
= & T_{1, j}+T_{2, j},
\end{aligned}
$$

where $\kappa_{j}:=k-j$. To calculate $T_{1, j}$, we consider the following finite sum:

$$
U_{j, v}:=\sum_{\substack{0<l_{1}<\cdots<l_{j} \\ 0<n_{1}<\cdots<n_{\kappa_{j}-v}<n_{\kappa_{j}-v+1} \\ l_{j}=n_{\kappa_{j}-v}}} \frac{1}{l_{1} \cdots l_{j} n_{1} \cdots n_{\kappa_{j}-v}} .
$$


By an argument similar to that of [5], we have

$$
\begin{aligned}
U_{j, v}= & \sum_{h=1}^{\min \left\{j, \kappa_{j}-v\right\}}\left(\begin{array}{c}
k-v-2 h \\
j-h
\end{array}\right) \\
& \times \sum_{\left(a_{1}, \ldots, a_{h}\right) \in \mathcal{S}_{h}^{k-v}} \zeta_{n_{\kappa_{j}-v+1}}\left(\{1\}_{a_{1}}, 2, \ldots,\{1\}_{a_{h}}, 2\right),
\end{aligned}
$$

where

$$
\zeta_{N}\left(r_{1}, \ldots, r_{k}\right):=\sum_{0<n_{1}<\cdots<n_{k}<N} \frac{1}{n_{1}^{r_{1}} \cdots n_{k}^{r_{k}}}
$$

is a finite sum. Consequently, we obtain

$$
\begin{aligned}
T_{1, j}= & \sum_{v=1}^{\kappa_{j}-1} \sum_{h=1}^{\min \left\{j, \kappa_{j}-v\right\}}\left(\begin{array}{c}
k-v-2 h \\
j-h
\end{array}\right) \\
& \times \sum_{\left(a_{1}, \ldots, a_{h}\right) \in \mathcal{S}_{h}^{k-v}} L(\underbrace{\{1\}_{a_{1}}, 2, \ldots,\{1\}_{a_{h}}, 2}_{k-v-h},\{1\}_{v} ; w_{-3} ; k-h, v) .
\end{aligned}
$$

Similarly,

$$
\begin{aligned}
& \text { (4.8) } \quad T_{2, j}=\sum_{v=1}^{\kappa_{j}} \sum_{h=1}^{\min \left\{j, \kappa_{j}-v+1\right\}}\left(\begin{array}{c}
k-v+1-2 h \\
j-h
\end{array}\right) \\
& \times \sum_{\left(a_{1}, \ldots, a_{h}\right) \in \mathcal{S}_{h}^{k-v+1}} L(\underbrace{\{1\}_{a_{1}}, 2, \ldots,\{1\}_{a_{h-1}}, 2,\{1\}_{a_{h}}}_{k-h-v},\{1\}_{v+1} ; w_{-3} ; k-h+1, v) .
\end{aligned}
$$

Combining (4.3), 4.5), 4.7) and (4.8), we have Theorem 2.1.

Acknowledgements. The author would like to express his gratitude to Professor Yasuo Ohno for his valuable comments. The author would also like to thank for a partial support of "Open Research Center" Project for Private Universities: matching fund subsidy from MEXT.

\section{References}

[1] H. Akatsuka, Zeta Mahler measures, J. Number Theory 129 (2009), 2713-2734.

[2] T. Arakawa and M. Kaneko, On multiple L-values, J. Math. Soc. Japan 56 (2004), 967-991.

[3] D. Boyd, Mahler's measure and special values of L-functions, Experiment. Math. 7 (1998), 37-82.

[4] C. Deninger, Deligne periods of mixed motives, $K$-theory and the entropy of certain $\mathbb{Z}^{n}$-actions, J. Amer. Math. Soc. 10 (1997), 259-281.

[5] N. Kurokawa, M. Lalín and H. Ochiai, Higher Mahler measures and zeta functions, Acta Arith. 135 (2008), 269-297. 
[6] C. J. Smyth, On measures of polynomials in several variables, Bull. Austral. Math. Soc. 23 (1981), 49-63.

[7] H. Tsumura, On some functional relations between Mordell-Tornheim double Lfunctions and Dirichlet L-functions, J. Number Theory 120 (2006), 161-178.

Yoshitaka Sasaki

Interdisciplinary Graduate School of Science and Engineering

Kinki University

Higashi-Osaka, Osaka 577-8502, Japan

E-mail: sasaki@alice.math.kindai.ac.jp

Received on 11.7.2009

and in revised form on 9.11.2009 